\title{
The Involvement of Endothelin Pathway in Chronic Psychological Stress-Induced Bladder Hyperalgesia Through Capsaicin-Sensitive C-Fiber Afferents
}

\author{
Chuying Qin', Yinhuai Wang', Sai Li², Yuanyuan Tang ${ }^{3}$, Yunliang Gao (D)' \\ 'Department of Urology, The Second Xiangya Hospital, Central South University, Changsha, 4I00II, People's Republic of China; ${ }^{2}$ Acupuncture and \\ Tuina School, Hunan University of Chinese Medicine, Changsha, 410208, People's Republic of China; ${ }^{3}$ Department of Oncology, The Second Xiangya \\ Hospital, Central South University, Changsha, 4I00II, People's Republic of China
}

Correspondence: Yunliang Gao, Department of Urology, The Second Xiangya Hospital, Central South University, No. I39. Renmin Road, Changsha, 4I00II, People's Republic of China, Email yunliang.gao@csu.edu.cn

Introductions: Interstitial cystitis/bladder pain syndrome (IC/BPS) is a poorly understood chronic disorder characterized by bladderrelated pain. Chronic psychological stress plays a key role in the exacerbation and development of IC/BPS via unclear mechanisms. This study aimed to investigate the role of endothelin 1 (ET-1) and its receptors in the development of chronic stress-induced bladder dysfunction.

Methods: Wistar-Kyoto rats were exposed to chronic (10 days) water avoidance stress (WAS) or sham stress, with subgroups receiving capsaicin pretreatment to desensitize C-fiber afferents. Thereafter, cystometrograms (CMG) were obtained with visceromotor response (VMR) simultaneously during intravesical saline or ET-1 infusion. CMG recordings were analyzed for the first and the continuous voiding cycles, respectively. Endothelin receptor type A (ETAR) expression was examined in the bladder tissues and L6-S1 dorsal root ganglions (DRGs). Toluidine blue staining was to check the bladder inflammation and double-labeling immunofluorescence (IF) staining was to identify the locations of ETAR, respectively.

Results: During saline infusion, WAS rats elicited significant decreases in pressure threshold (PT) and in the ratio of VMR threshold/ maximum intravesical pressure (IVPmax), and a significant increase in VMR duration and area under the curve (AUC). ET-1 infusion induced similar alternations in WAS rats, but further significantly diminished the pressure to trigger PT and VMR, together with a more forceful and longer VMR. The sole effect of WAS exposure or ET-1 administration on the micturition reflex could be suppressed by capsaicin pretreatment. WAS exposure significantly induced an increased number of total mast cells in the bladder, while capsaicin pretreatment possibly antagonized them. No significant difference in ETAR expression was found between all groups. IF staining indicated the co-localization of ETAR and calcitonin gene-related peptides in both bladder and DRGs.

Conclusion: The activation of ET-1 receptors could enhance chronic stress-induced bladder hypersensitization and hyperalgesia through capsaicin-sensitive C-fiber afferents. Targeting the endothelin pathway may have therapeutic value for IC/BPS.

Keywords: endothelin, endothelin receptor type A, interstitial cystitis, pain, psychological stress, voiding dysfunction

\section{Introduction}

Interstitial cystitis/bladder pain syndrome (IC/BPS) is a chronic debilitating condition characterized by bladder-related pelvic pain and lower urinary tract (LUT) symptoms. ${ }^{1}$ Due to the heterogeneity in definitions, the true prevalence is difficult to define but is estimated to $2.7-6.5 \%$ in women and $1.9-4.2 \%$ in men. ${ }^{2,3}$ IC/BPS patients usually suffer from a detrimental quality of life and exaggerated medical burden. The bladder-related pain in IC/BPS patients remains the key issue imperative to be solved. Despite great progress made in recent years, the exact etiology of IC/BPS is still enigmatic and none of therapeutic applications is curative. Recently, accumulative studies have revealed the key role of chronic psychological stress in exacerbation and development of IC/BPS. Patients with IC/BPS have displayed a high prevalence of comorbid stress-related affective disorders such as anxiety and depression. ${ }^{4,5}$ And depression may be directly related 
to pain, but not urinary symptoms. ${ }^{6}$ Further investigation of the pathophysiology of chronic stress-induced bladder dysfunction may lead to the effective treatment of patients with IC/BPS.

To explore the interplay of chronic stress and IC/BPS, we have conducted an animal model of chronic water avoidance stress (WAS). WAS, a well-studied stress model, appears to reflect the stress variability seen in patients and mimic the key features of urinary frequency and bladder hyperalgesia experienced by IC/BPS patients. ${ }^{7-12}$ WAS could induce significant changes at multiple levels of micturition pathway, including bladder histopathological alterations ${ }^{13-15}$ and spinal glutamate neurotransmission dysfunctions. ${ }^{9,16}$ By using this WAS model, several drugs were tested to treat voiding dysfunctions such as quercetin,${ }^{15}$ taurine, ${ }^{17}$ epigallocatechin gallate, ${ }^{18}$ cyclooxygenase-2 inhibitor, ${ }^{19}$ silodosin ${ }^{20}$ and guanethidine. ${ }^{21}$ In addition to them, our group's previous work also showed WAS-induced bladder inflammatory alternations such as increased vascularity and mast cells. ${ }^{7}$ Recently we indicated that WAS-induced bladder sensation and nociceptive response may be related to the sensitized bladder C-fiber afferent and the increased engagement of portions of the micturition circuit. ${ }^{10,12}$ Sensitization and enhanced excitability of bladder C-fiber afferents, possibly as a result of inflammation, is likely to be involved in the pathophysiological basis of IC/BPS in particular pain. ${ }^{22}$ However, the mechanisms underlying chronic stressinduced hyperexcitability of C-fiber afferents in IC/BPS have still not been definitively identified.

Endothelin-1 (ET-1), a potent vasoconstrictor peptide, exerts pleiotropic biological functions by binding to two different $G$ protein-coupled receptors (endothelin receptor type A, ETAR; endothelin receptor type B, ETBR). These binding receptors have been identified in the neural and non-neural LUT components including bladder tissues and peripheral nerve system, where they are primarily of the ETAR subtype. ${ }^{23}$ ET-1 and its receptors play important roles in regulating bladder contraction and sensation under normal and pathological conditions. ${ }^{24,25}$ For instance, by action on ETAR, ET-1 could induce detrusor overactivity and modulate sensory function in the peripheral nervous system through C-fibers. ${ }^{25} \mathrm{~A}$ just-published study revealed the involvement of endothelin pathway in urinary urgency and incontinence. ${ }^{26}$ Particularly, ET-1 is a known powerful algogen and increases nerve sensitization and painful feeling in humans and spontaneous nociceptive behaviors in animals via ETAR. ${ }^{27}$ The activation of ETAR also enables to increase dorsal root ganglion (DRG) neuronal excitation. ${ }^{28-30}$ Moreover, endothelin pathway shows a close link to different inflammatory conditions such as bladder inflammation. ${ }^{31,32}$ Under chronic or repeated exposure to psychological stress could lead to endothelin system dysfunction via ETAR pathway. ${ }^{33,34}$

Therefore, it seems appropriate to posit that endothelin pathway may play a key role in the chronic psychological stress-induced bladder hyperalgesia of IC/BPS. As yet, to our knowledge, no studies have demonstrated the relationship among endothelin pathway, chronic stress and IC/BPS. In present study, we employed WAS model to explore the effects of ET-1 application on the bladder function after stress exposure and address whether endothelin receptors activation mediating the effects through bladder $\mathrm{C}$ fiber afferents. The location and expression level of probably involved ETAR subtype was also been analyzed.

\section{Materials and Methods}

\section{Animals and Classification}

Adult female Wistar Kyoto (WKY) rats (180-200 g) were provided by Charles River Laboratories (Beijing, China). WKY strain was selected due to its genetic predisposition to anxiety. ${ }^{35}$ Rats were housed under standard conditions (12:12-hour light-dark cycle) with water and standard chow ad libitum. All purchased rats had an adjustment period of 1-2 weeks prior to the experiment. All experimental procedures were approved by the Institutional Animal Care and Use Committee at Second Xiangya Hospital of Central South University (No.2019001) and were performed in strict accordance with the standards in the NIH Guide for the Care and Use of Laboratory Animals. The grouping details were listed in Table 1.

\section{Chronic WAS Protocol}

24 rats were randomly divided into Sham group $(n=12)$ and WAS group $(n=12)$. The average body weight at baseline was no significant difference (197.3 \pm 13.0 vs $199.2 \pm 12.7 \mathrm{~g}, \mathrm{p}>0.05)$. WAS protocol has been previously described in detail. ${ }^{10,12,16}$ Briefly, the rat was placed on a platform centered on the floor of a plastic container. The container was filled with room temperature water $1 \mathrm{~cm}$ below the top of the platform. Rats were subjected to WAS for 1 hour per day 
Table I Groups of Rats Used in the Present Study

\begin{tabular}{|l|l|l|l|l|l|}
\hline Group Number & Group Name & Number of Rats & Pretreatment & Exposure & Infusion Solution \\
\hline I & WAS & 12 & None & WAS & Ist Saline, 2nd ET-I \\
2 & Sham & 12 & None & Handling & Ist Saline, 2nd ET-I \\
3 & WAS-Cap & II & Capsaicin & WAS & Ist Saline, 2nd ET-I \\
4 & Sham-Cap & II & Capsaicin & Handling & Ist Saline, 2nd ET-I \\
\hline
\end{tabular}

Abbreviations: ET-I, endothelin I; WAS, water avoidance stress.

for 10 consecutive days. Rats in Sham group were exposed to handling only for 1 minute at the beginning and end of the experimental hour. The above procedures were done in the morning to minimize circadian effects.

\section{Capsaicin Pretreatments}

Other 22 WKY rats were pretreated with capsaicin (Sigma-Aldrich, MO, USA) and separated to Sham-Cap group $(\mathrm{n}=11)$ and WAS-Cap group $(\mathrm{n}=11)$. The baseline in body weight was $195.4 \pm 5.5 \mathrm{~g}$ vs $193.8 \pm 12.4 \mathrm{~g}(\mathrm{p}>0.05)$. Capsaicin pretreatment was to desensitize C-fibers afferents using previously described methods. ${ }^{36-38}$ In brief, capsaicin was dissolved in a vehicle containing 10\% ethanol, 10\% Tween 80 (Sigma-Aldrich) and 80\% saline at a concentration of $10 \mathrm{mg} / \mathrm{mL}$. Capsaicin solutions were administered subcutaneously over a 36-hour period (total dose $125 \mathrm{mg} / \mathrm{kg})$. To prevent capsaicin-mediated respiratory failure, atropine $(0.5 \mathrm{mg} / \mathrm{kg})$ were injected intraperitoneally before the capsaicin injection. The eye wipe test was utilized to evaluate capsaicin desensitization before WAS or handling exposure.

\section{Surgical Procedures}

One day after completion of the WAS protocol, the rats were anesthetized by intraperitoneal injection of $1.2 \mathrm{~g} / \mathrm{kg}$ urethane (Sigma-Aldrich) 1 hour before surgery and then placed on a $37^{\circ} \mathrm{C}$ water-circulating heating pad. For recording the visceromotor response (VMR), a pair of fine and insulated silver wire electrodes (A-M Systems, WA, USA) with exposed tips were embedded into the left abdominal external oblique muscle. VMR is well established and validated as a quantitative measure of visceral nociception. ${ }^{10,39,40}$ VMR wires were connected to the data acquisition system. A polyethylene 50 catheter was inserted into the bladder through the urethra outlet. The catheter was connected via a 3-way connector to a syringe pump (Advance 1200, CellPoint Scientific, MD, USA) and a pressure transducer (Biopac Systems, CA, USA) for monitoring intra-bladder pressure. ${ }^{8,41}$ Cystometrogram (CMG) and VMR were all obtained by using a biological signal acquisition system (MP150, Biopac Systems) (Figure 1).

\section{Recordings of CMG and VMR}

CMG and VMR were recorded simultaneously during intravesical infusion in all groups. ${ }^{10}$ After one hour of urethane anesthesia, normal saline $(0.9 \% \mathrm{NaCl})$ with $0.05 \%$ bovine serum albumin (Sigma-Aldrich) was infused continuously through the catheter at a rate of $6 \mathrm{~mL} / \mathrm{h}$. The bladder infusion would stop after obtaining three stable voiding cycles for analysis. The bladder was then emptied and allowed 20-minute rest periods. Next, the process for recording CMG and VMR was repeated by infusing ET-1 solution ( $1 \mu \mathrm{M}$, Sigma-Aldrich), which was prepared by dissolving ET-1 into $0.9 \%$ saline with $0.05 \%$ bovine serum albumin.

The analysis of recording of CMG and VMR were consisted of two parts, the first voiding cycle and the continuous stable voiding cycles, to achieving a more comprehensive analysis. For the analysis of first voiding cycle, the latency of the first void/leak (LFV) was measured as the latency from the beginning of bladder filling to occurrence of the first void/ leak. The bladder capacity $(\mathrm{mL})$ was calculated as LFV $(\mathrm{min}) \times$ infusion rate $(0.1 \mathrm{~mL} / \mathrm{min})$. The pressure threshold $(\mathrm{PT})$ to first void/leak, the maximum intravesical pressure (IVPmax) and the ratio of VMR threshold/IVPmax were obtained. The VMR threshold pressure was determined as the bladder pressure evoking VMR. If VMR was not evoked during voiding, the ratio of VMR threshold/IVPmax would be treated as $100 \%$. 
A Water avoidance stress
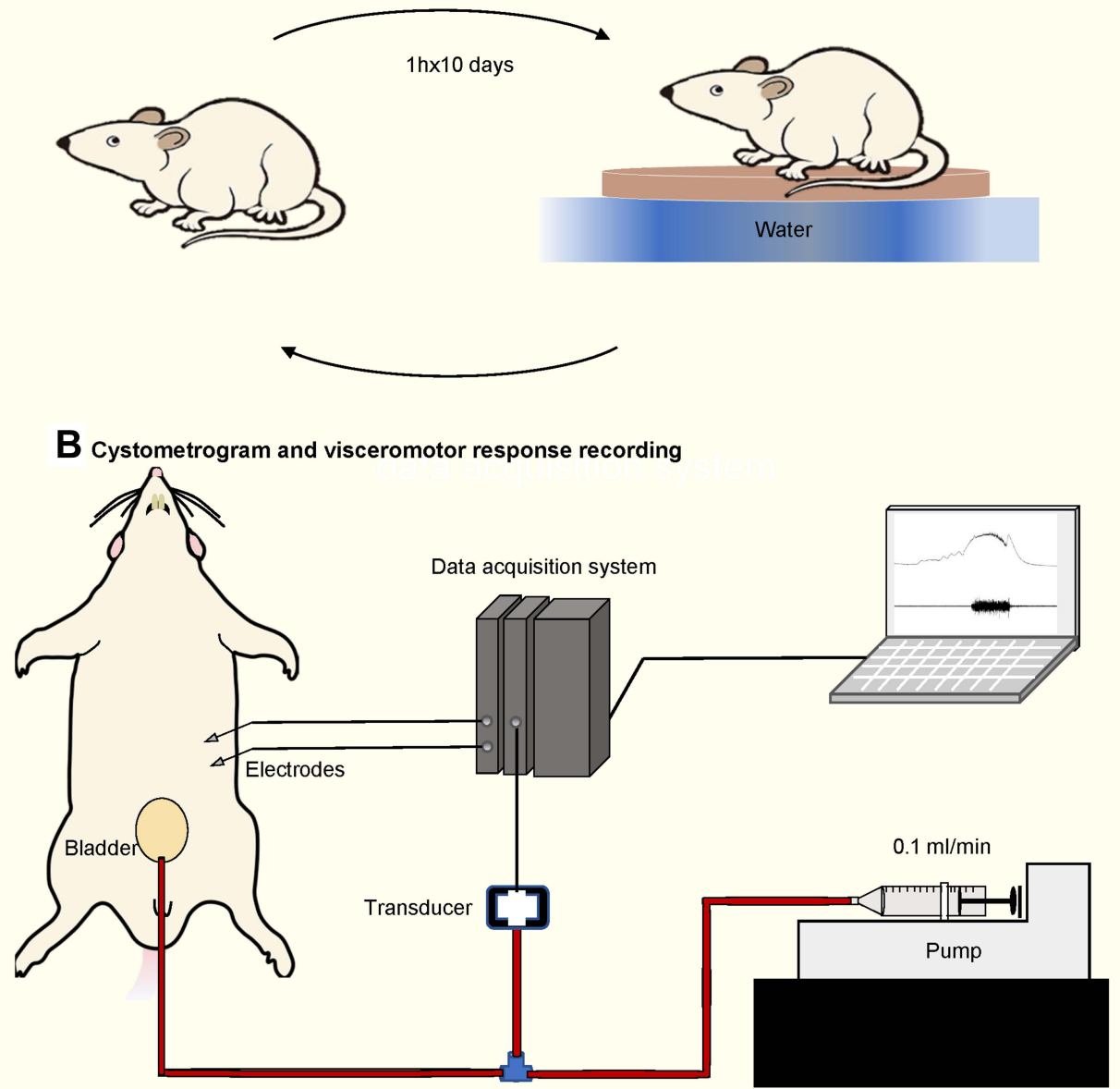

Figure I Water avoidance stress protocol and cystometry setup. (A) Schematic drawing of the performing water avoidance stress to animals. (B) Schematic drawing of the cystometry setup. A pair of electrodes were embedded into the left abdominal external oblique muscle. A syringe pump is connected to a pressure transducer, which is connected to the intravesical catheter.

For the analysis of continuous voiding cycles, contraction duration (CD), inter contraction interval (ICI), PT, IVPmax and the ratio of VMR/IVPmax were calculated from three consecutive voiding cycles. In the same voiding cycle, the duration, amplitude and area under curve (AUC) measurements for the VMR firing activity were also calculated. Notably, if the VMR did not occur during voiding, the AUC and duration of VMR were regarded as zero. All parameters were analyzed using Acqknowledge software (Biopac Systems).

\section{Tissue Preparation}

After CMG recording, all rats were deeply anesthetized with intraperitoneal injection of urethane $(5 \mathrm{~g} / \mathrm{kg})$. Bladders and L6-S1 DRGs were dissected out and then flash frozen by liquid nitrogen. The samples were stored in liquid nitrogen for future Western blot and/or RT-qPCR analysis.

Other 4 rats per each group (total 16) were exempted from CMG analysis to obtain bladder samples for staining, while naïve DRGs for staining were only harvested from two no capsaicin-pretreated rats among them. After dissection, tissues were fixed in $4 \%$ paraformaldehyde, embedded in paraffin, cut into sections (4- $\mu$ m thickness) and mounted on coated slides. Slides were dried and stored for future staining analysis. 


\section{Western Blot Analysis}

Western blot analysis was carried out for the detection ETAR protein levels. Bladder tissues were thoroughly grinded under liquid nitrogen and protein samples were extracted using RIPA buffer with $1 \mathrm{mM}$ PMSF (Beyotime Biotech, Shanghai, China). BCA Kit (Beyotime Biotech, China) was used to measure protein concentration. Sodium dodecyl sulphate-polyacrylamide gel was applied to analyze $30 \mu \mathrm{g}$ of protein from each group and then transferred to a polyvinylidene fluoride membrane. After the primary antibodies ETAR (ab85163, Abcam, MA, USA) and GAPDH (60004-1-Ig, Proteintech, Wuhan, China) and appropriate Secondary antibody reactions, the blot membranes were processed by using an enhanced chemiluminescence (ECL) kit (Immobilon, Sigma-Aldrich), followed by film exposure and densitometry analysis.

\section{qRT-PCR Analysis of mRNA}

qRT-PCR was employed to estimate ETAR gene expression. Total RNA of bladder tissue was extracted by using Trizol reagent (Ambion, Carlsbad, USA), while total RNA of DRGs was extracted by using the RNeasy Micro Kit (Qiagen, USA) following instructions. Reverse transcription kit and real-time PCR kit were purchased from Takara Biotechnology (China) and performed according to the manufacturer's instruction. Relative quantification was analyzed using the 2 $-\Delta \Delta \mathrm{Ct}$ method. The primers in this study were referred to previous studies ${ }^{42}$ and purchased from Sangon (Shanghai, China) and were indicated in Table 2.

\section{Toluidine Blue Staining}

Toluidine blue staining was conducted to verify the bladder inflammation condition as previous study. ${ }^{7}$ The slides with bladder tissues were underwent staining with toluidine blue reagent (Servicebio, Wuhan, China). In each bladder crosssectional specimen, total number of mast cells were counted under a light microscope (Nikon, Tokyo, Japan). Three sections were counted for each bladder specimen. The mean number of mast cells per bladder area in $\mathrm{mm}^{2}$ was calculated and compared.

\section{Double-Labeling Immunofluorescence Staining}

ETAR was double-labeled with calcitonin gene-related peptides (CGRP) in the bladder and L6 DRGs. CGRP is a type of neurotransmitter related to peripheral nociceptors and stored in a population of capsaicin-sensitive C-fiber afferents. Immunofluorescence (IF) staining was conducted using standard techniques from Servicebio. Brief, IF was performed by incubating the paraffin sections with primary ETAR antibody (sc-518060, Santa Cruz, TX, USA) and CGRP antibody (PC205L, Sigma-Aldrich), which were incubated with Cy3 conjugated Goat Anti-mouse $\operatorname{IgG}(\mathrm{H}+\mathrm{L})$ and FITC conjugated Goat Anti-Rabbit IgG $(\mathrm{H}+\mathrm{L})$, respectively. 4',6-diamidino-2-phenylindole (DAPI, Servicebio) was used for nuclear staining. The immunoreactions in the bladder and DRGs were observed using a confocal microscope.

Table 2 Nucleotide Sequences of Primers for qRT-PCR

\begin{tabular}{|l|l|l|}
\hline Name & Primer Notation & Sequence \\
\hline GAPDH & Forward & GCATCCTGCACCACCAACTG \\
& Reverse & GCCTGCTTCACCACCTTCTT \\
ETAR & Forward & CTCAGCGAACACCTCAAGCA \\
& Reverse & GGCTTAAGTGAAGAGGGAACCA \\
\hline
\end{tabular}

Abbreviations: ETAR, endothelin receptor type A; GAPDH, glyceraldehyde-3-phosphate dehydrogenase. 


\section{Data Analysis}

Values are presented as means \pm standard deviation (SD). Student's $t$ test, Mann-Whitney test and one-way ANOVA test were used for statistical analysis as appropriate. A statistical difference was considered when a $p<0.05$. All data were statistically analyzed using GraphPad Prism version 9 (GraphPad Software, Inc. San Diego, USA).

\section{Results}

\section{CMG and VMR Recordings of First Voiding Cycle During Saline Infusion}

During the first voiding cycle under saline infusion (Figure 2A-E), WAS group rats showed significant decreases in PT $\left(10.8 \pm 3.5\right.$ vs $\left.16.1 \pm 5.8 \mathrm{cmH}_{2} \mathrm{O}, \mathrm{p}<0.05\right)$ and the ratio of VMR threshold/IVPmax (54.9 \pm 10.7 vs $\left.71.4 \pm 12.6 \%, \mathrm{p}<0.05\right)$. WAS did not appear to alter IVPmax and bladder compacity when compared to Shams.

After capsaicin pretreatment, WAS-Cap rats compared to Sham-Cap also showed a significant decrease in PT (10.5 \pm 3.0 vs $\left.15.0 \pm 6.5 \mathrm{cmH}_{2} \mathrm{O}, \mathrm{p}<0.05\right)$ and IVPmax $\left(26.3 \pm 3.4\right.$ vs $\left.30.9 \pm 4.4 \mathrm{cmH}_{2} \mathrm{O}, \mathrm{p}<0.05\right)$ but no significant differences in the ratio of VMR threshold/IVPmax and capacity. In comparison with no capsaicin-pretreated (Sham and WAS) animals, capsaicin administration (Sham-Cap and WAS-Cap) could increase the ratio of VMR threshold/IVPmax but decrease the IVPmax.

\section{CMG and VMR Recordings of Continuous Voiding Cycles During Saline Infusion}

The continuous voiding results during saline filling were in according with those of first voiding cycle (Figure $3 \mathrm{~A}-\mathrm{G}$ ). A lower PT $\left(8.6 \pm 1.5\right.$ vs $\left.10.9 \pm 3.6 \mathrm{cmH}_{2} \mathrm{O}, \mathrm{p}<0.05\right)$ triggering voiding cycles was indicated in WAS rats than these in Sham ones. The ratio of VMR threshold/IVPmax also showed the VMR to appear significantly earlier in WAS animals compared to Shams $(45.2 \pm 7.6$ vs $57.1 \pm 16.8 \%, \mathrm{p}<0.05)$. A slightly increased IVPmax was found in WAS rats rather than Shams. Particularly, WAS rats presented a longer VMR duration $(21.7 \pm 9.6$ vs $14.9 \pm 5.8$ second, $\mathrm{p}<0.05)$ and a larger VMR AUC $(0.6 \pm 0.3$ vs $0.3 \pm 0.2 \mathrm{mV}$-second, $\mathrm{p}<0.05)$ as compared to Sham ones. There was no significant difference in ICI between WAS and Sham groups ( $398.5 \pm 160$ vs $418.2 \pm 166.0$ second, $\mathrm{p}>0.05)$. No significant changes appeared in other CMG parameters (CD) or VMR amplitude between WAS and Sham rats.

After capsaicin pretreatment, WAS-Cap rats presented no significant differences in ICI compared to Sham-Cap ones (444.3 \pm 175.6 vs $395.9 \pm 147.2$, p >0.05). Besides, other CMG parameters (PT, VMR threshold/IVPmax ratio, IVPmax, $\mathrm{CD}$ ) or VMR parameters (amplitude, duration and AUC) showed no significant differences between WAS-Caps and Sham-Caps. In addition, capsaicin pretreatment (Sham-Cap and WAS-Cap) significantly increased the ratio of VMR threshold/IVPmax but reduced IVPmax compared to those without pretreatment (Sham and WAS). Significant reductions in VMR amplitude, duration and AUC were also noted in capsaicin-pretreated rats.

\section{CMG and VMR Recordings of First Voiding Cycle During ET-I Infusion}

During the first voiding cycle under ET-1 infusion (Figure 4A-E), both Sham and WAS rats showed further decreases in PT and the ratio of VMR threshold/IVPmax ratio when compared with saline infusion. However, these two indicators, together with IVPmax and bladder capacity, reported no significant differences between WAS and Sham groups.

Between Sham-Cap group and WAS-Cap group no significant differences were found in CMG and VMR parameters. However, a significantly reduced IVPmax still could be found in capsaicin-treated rats when compared to non-capsaicintreated rats.

\section{CMG and VMR Recordings of Continuous Voiding Cycles During ET-I Infusion}

ET-1 infusion (Figure 5A-G) in WAS rats further significantly decreases in PT $\left(7.9 \pm 1.1\right.$ vs $\left.10.2 \pm 3.5 \mathrm{cmH}_{2} \mathrm{O}, \mathrm{p}<0.05\right)$ and in the ratio of VMR threshold/IVPmax $(34.3 \pm 15.7$ vs $48.7 \pm 16.4 \%, \mathrm{p}<0.05)$ compared to Shams. Differences of PT and the ratio of VMR threshold/IVPmax appeared to be increased during ET-1 infusion as compared to saline infusion. Moreover, ET-1 induced a further longer $(30.2 \pm 22.8$ vs $14.8 \pm 6.3$ second, $\mathrm{p}<0.05)$ and more forceful $(1.0 \pm 0.7$ vs $0.4 \pm 0.3$ $\mathrm{mV}$-second, $\mathrm{p}<0.05$ ) VMR in WAS rats in comparison with Shams. Instead, ET-1 could not induce significant change of 

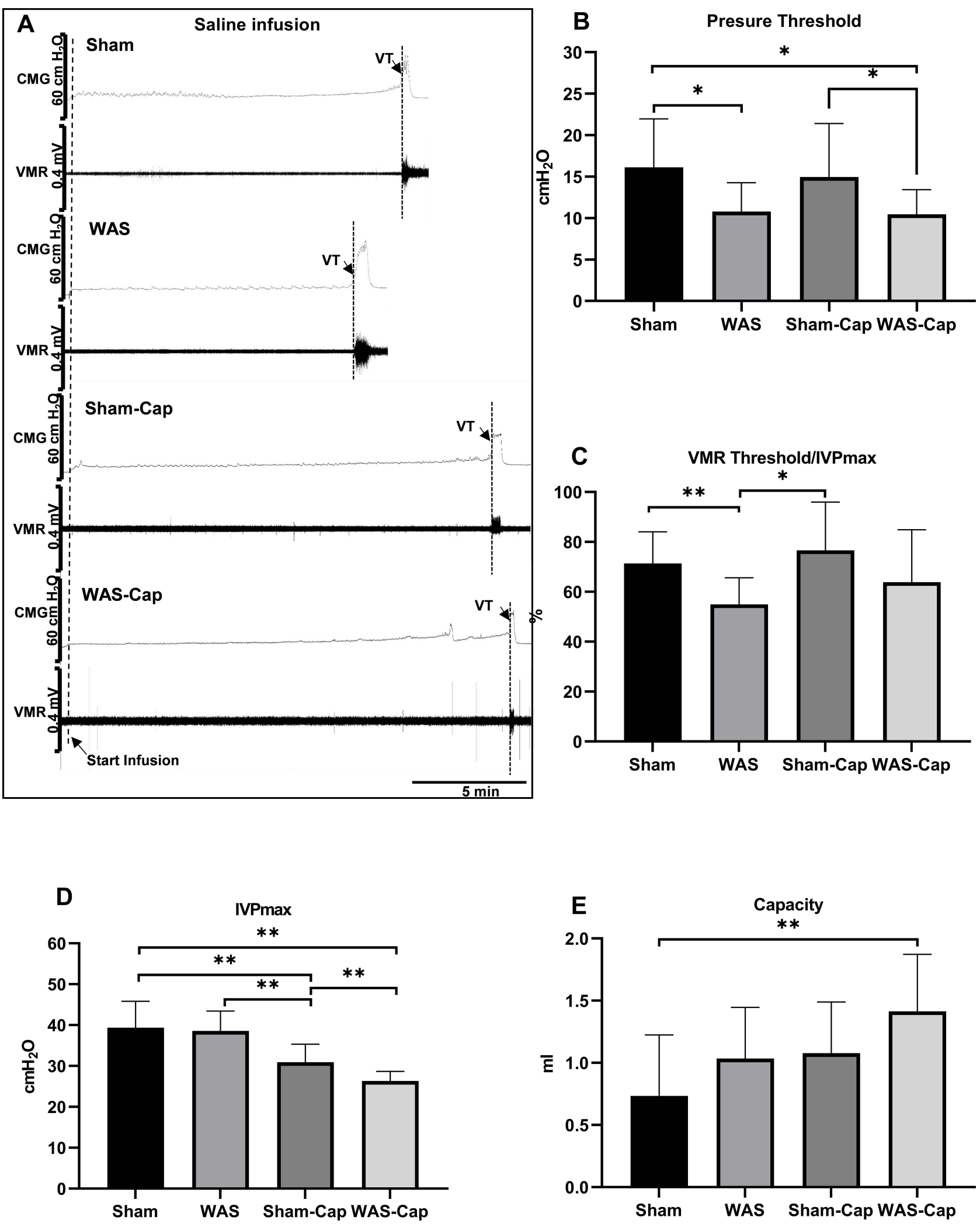

Figure 2 Cystometry and visceromotor response (VMR) recordings of first voiding cycle during saline infusion. (A) Representative recording examples of each group. (B) Alternations in pressure threshold. (C) Alternations in the ratio of VMR threshold/maximum intravesical pressure (IVPmax). (D) Alternations in IVPmax. (E) Alternations in bladder capacity. VT, VMR threshold. *Represents $\mathrm{p}<0.05$. **Represents $\mathrm{p}<0.01$. 

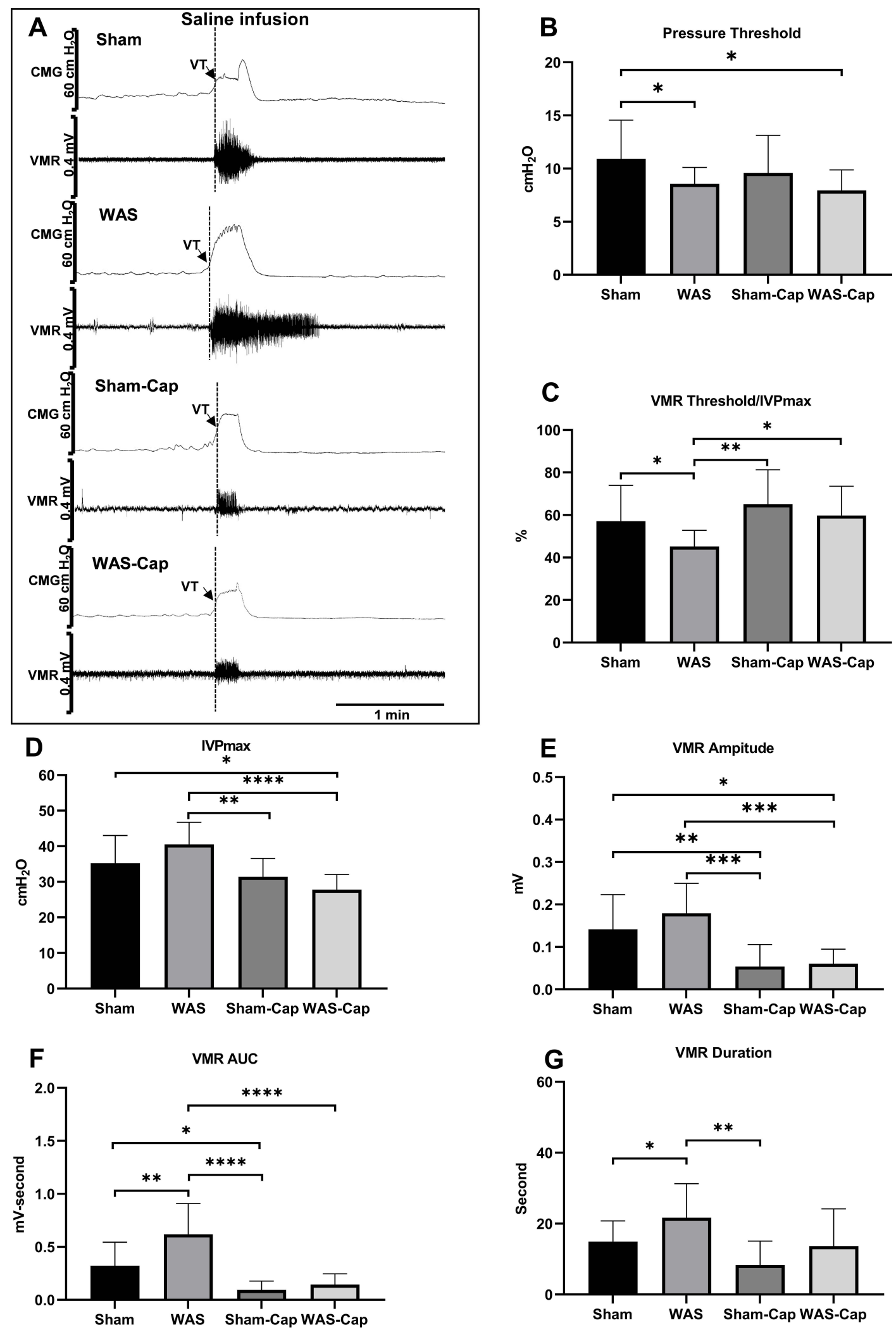

Figure 3 Cystometry and visceromotor response (VMR) recordings of continuous voiding cycles during saline infusion. (A) Representative recording examples of each group. (B) Alternations in pressure threshold. (C) Alternations in the ratio of VMR threshold/maximum intravesical pressure (IVPmax). (D) Alternations in IVPmax. (E) Alternations in the amplitude of VMR. (F) Alternations in the area under the curve (AUC) of VMR. (G) Alternations in the duration of VMR. VT, VMR threshold. $*$ Represents $p<0.05$. **Represents $p<0.01$. ***Represents $p<0.001$. ****Represents $p<0.0001$. 


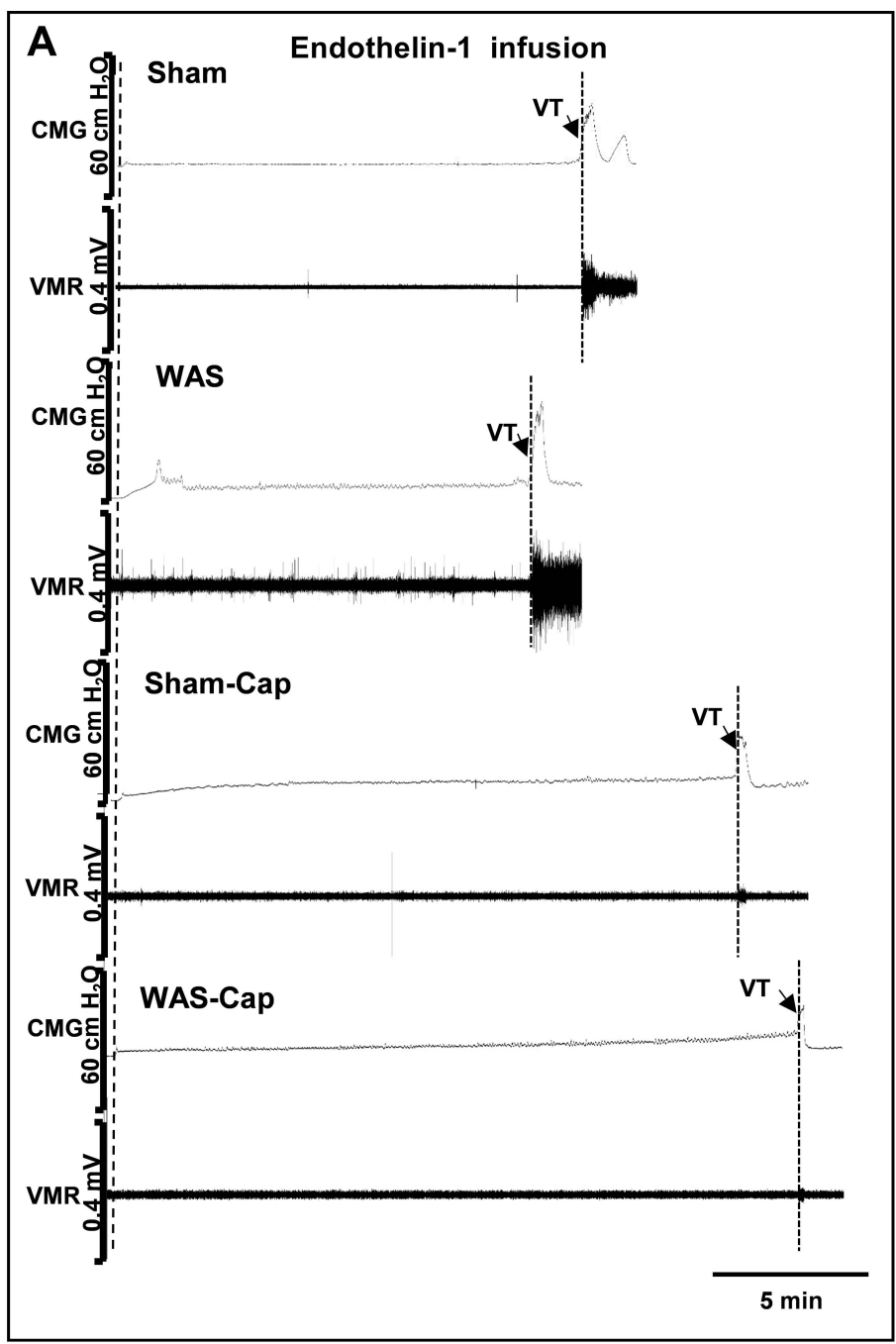

B

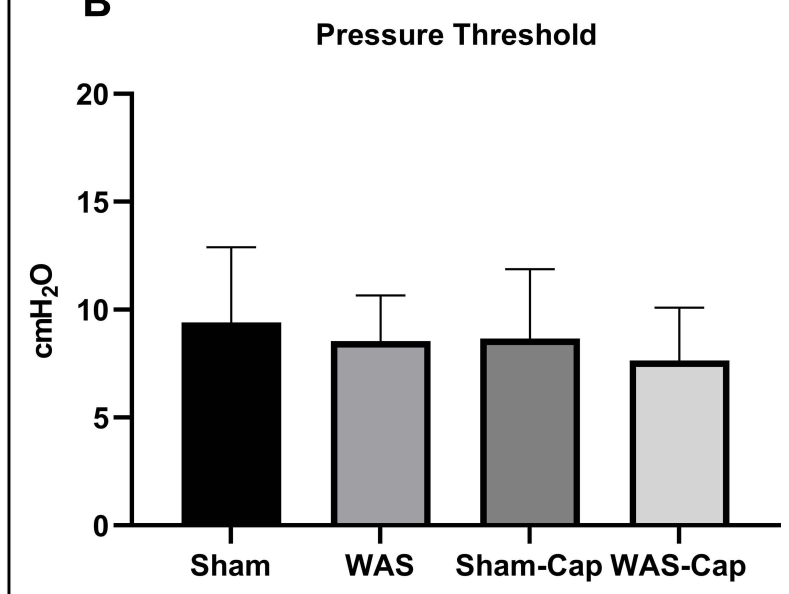

C VMR Threshold/IVPmax
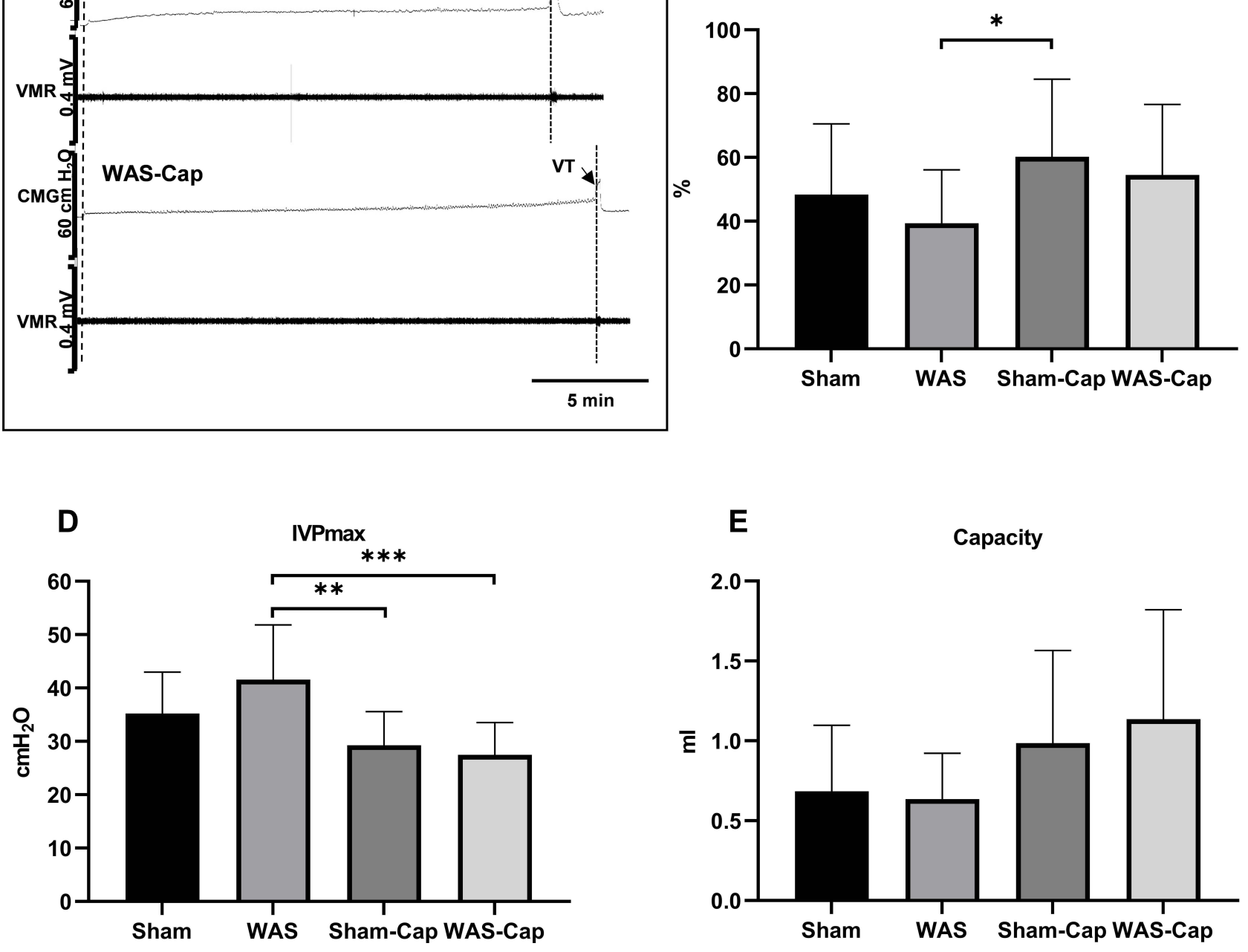

Figure 4 Cystometry and visceromotor response (VMR) recordings of first voiding cycle during endothelin-I infusion. (A) Representative recording examples of each group. (B) Alternations in pressure threshold. (C) Alternations in the ratio of VMR threshold/maximum intravesical pressure (IVPmax). (D) Alternations in IVPmax. (E) Alternations in bladder capacity. VT, VMR threshold. * represents $\mathrm{p}<0.05$. **Represents $\mathrm{p}<0.01$. ***Represents $\mathrm{p}<0.00 \mathrm{I}$. 

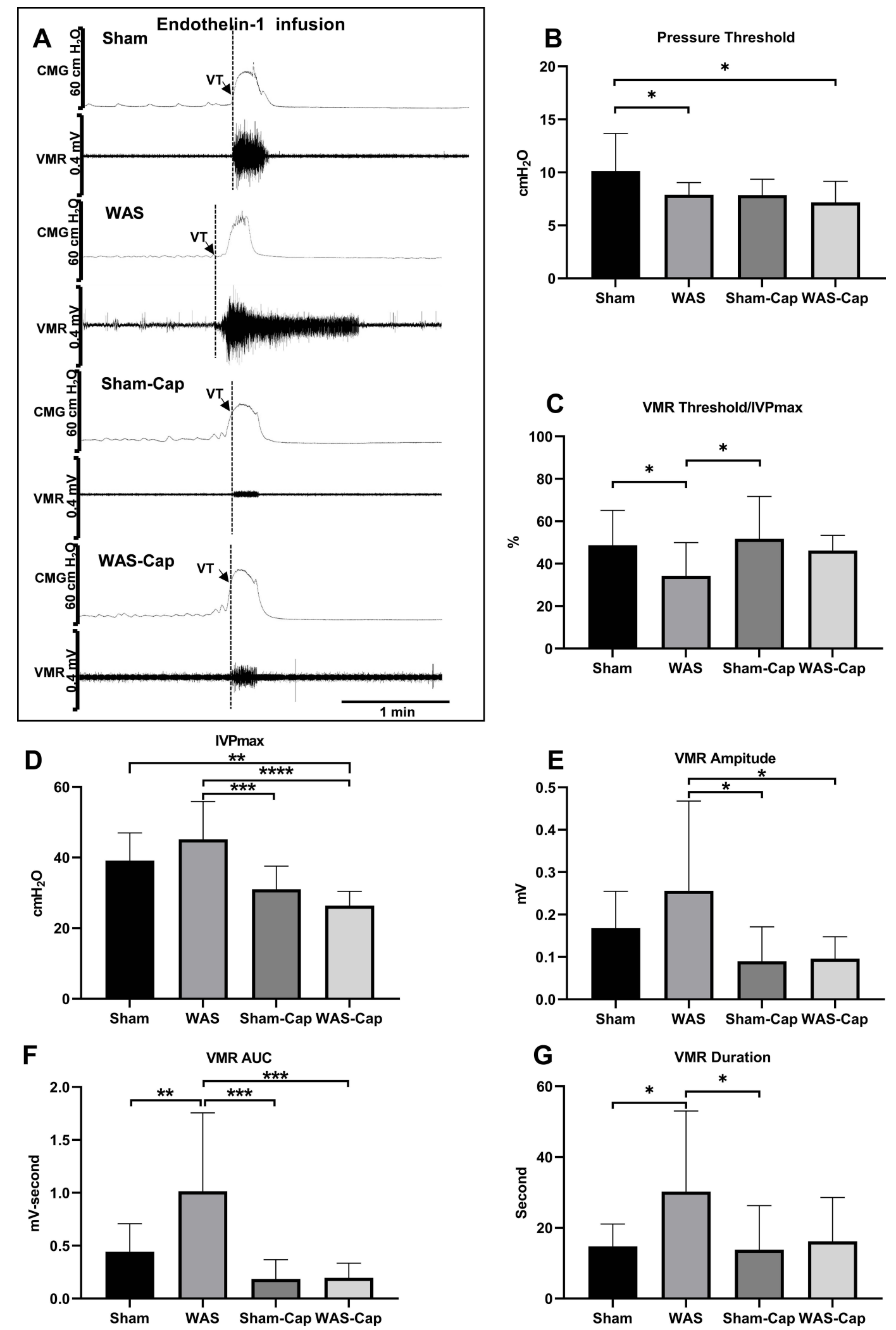

Figure 5 Cystometry and visceromotor response (VMR) recordings of continuous voiding cycles during saline infusion. (A) Representative recording examples of each group. (B) Alternations in pressure threshold. (C) Alternations in the ratio of VMR threshold/maximum intravesical pressure (IVPmax). (D) Alternations in IVPmax. (E) Alternations in the amplitude of VMR. (F) Alternations in the area under the curve (AUC) of VMR. (G) Alternations in the duration of VMR. VT, VMR threshold. $*$ Represents $p<0.05$. **Represents $p<0.01$. ***Represents $p<0.001$. ****Represents $p<0.0001$. 
ICI between WAS and Sham rats (317.2 \pm 154 vs 302.1 \pm 186.2 ). No significant alternations were reported in other CMG parameters (CD) or VMR amplitude between WAS and Sham groups.

Capsaicin pretreatment further increased ICI in both WAS-Cap and Sham-Cap rats (404.4 \pm 134.2 vs $406.7 \pm 184.5$, $\mathrm{p}>0.05$ ). In addition, capsaicin pretreatment diminished the differences in other CMG and VMR parameters between Sham-Cap and WAS-Cap. Consistent with the results of continuous voiding cycles during saline infusion, capsaicin pretreatment (Sham-Cap and WAS-Cap) significantly reduced IVPmax, VMR amplitude and VMR AUC compared to non-capsaicin-treated ones (Sham and WAS).

\section{The Altered Expression Level of ETAR in the Bladder and DRG}

ETAR mRNA was expressed in the bladder and L6-S1 DRGs (Figure 6A-D). However, no statistically significant difference was found in ETAR protein and mRNA expressions between these four groups.

\section{Toluidine Blue Staining}

Our toluidine blue staining (Figure 7A-E) demonstrated that bladders of WAS animals showed an increased total mast cells compared to Sham ones, which could be attenuated by capsaicin pretreatment.

\section{Double-Labeling if Staining}

The immunoreactivity of ETAR was positive in the bladder and L6 DRG neurons (Figure 7F-N). Double-labeling staining also revealed that CGRP-immunoreactive sensory nerve terminals co-expressed ETAR beneath the bladder epithelium as well as bladder muscle. Consistently, ETAR was also co-localized in CGRP-positive DRG neurons in particular small-to-medium size ones.

\section{Discussion}

Chronic psychological stress enables to influence the development or exacerbation of symptoms in IC/BPS via as yet unclear mechanisms. By conducting WAS model, we here revealed for the first time that the activation of endothelin receptors (ETAR probably) in capsaicin-sensitive C-fiber afferents could increase bladder hypersensitivity and hyperalgesia after chronic stress exposure (Figure 8). Current findings suggested the possibility of endothelin pathway as a novel therapeutic target for IC/BPS.

Functionally, this study demonstrated bladder hypersensitivity and hyperalgesia after WAS exposure. As shown by CMG recordings, WAS rats reported a decreased PT during saline infusion when compared with Shams. To wit, a lower pressure can trigger the voiding phase, suggesting the appearance of WAS-induced bladder hypersensitivity. Moreover, WAS rats showed a VMR that appeared earlier and more forcefully than that in Shams under saline infusion, manifested by a lower ratio of VMR threshold/IVPmax but a larger AUC of VMR. Given VMR as a reliable and quantitative marker for visceral nociception, ${ }^{10,39,40}$ it was reasonable to assume that WAS exposure may alter the properties of afferent sensory pathway with a decreased pain threshold but an increased pain input. In addition, a longer VMR duration in WAS rats represented a longer sensory/pain response to bladder stimuli. These results were consistent with our previous studies, which documented chronic stress-induced bladder functional changes particularly bladder hyperalgesia. ${ }^{10,12,16}$ Additionally, our prior brain mapping analysis implicated that WAS could increase engagement of portions of the micturition circuit responsive to urgency and viscerosensory perception. ${ }^{12,16}$ The increased functional brain activation in WAS rats, to a certain extent, may arise from the increased bladder afferent sensory input. These WAS-induced alterations were in line with other IC/BPS reports using different stressors such as neonatal maternal separation stress $^{39}$ and social stress, ${ }^{43}$ supporting the sensitization and hyperexcitability of bladder afferent pathways as one of the pathophysiological basis of stress-related IC/BPS.

Particularly, we further verified our previous finding that sensitized C-fiber afferents were possibly engaged in the facilitatory effect of stress on bladder sensory information processing. ${ }^{10} \mathrm{C}$-fiber afferents are small and unmyelinated fibers with specific functions of signaling inflammatory or noxious events in the bladder. ${ }^{44}$ After capsaicin pretreatment, there was no significant difference in PT and the ratio of VMR threshold/IVPmax between WAS-Cap and Sham-Cap groups during saline infusion. The duration, amplitude and AUC of VMR all showed no significant difference between 
A

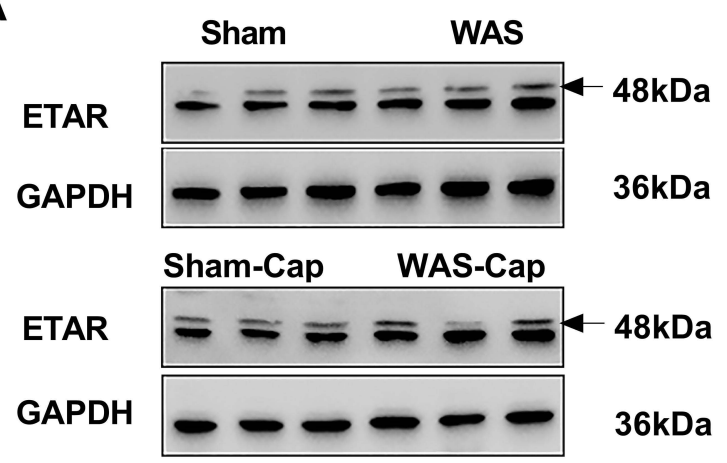

ETAR proteins expression in bladder

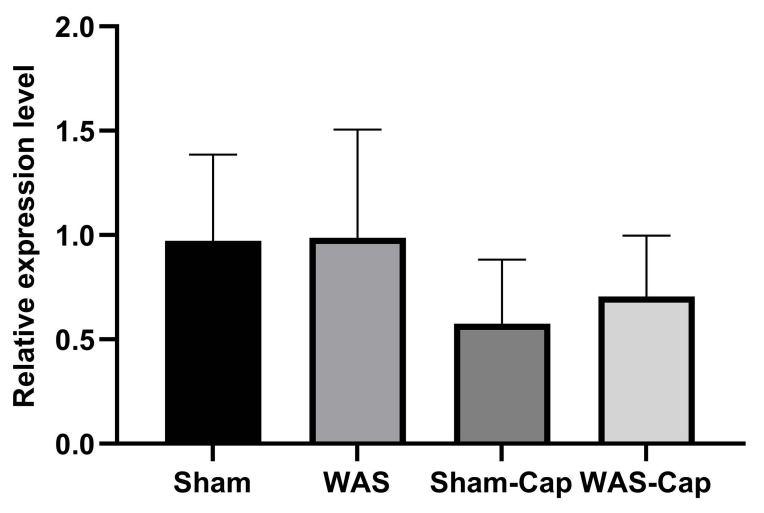

B ETAR mRNA expression in bladder

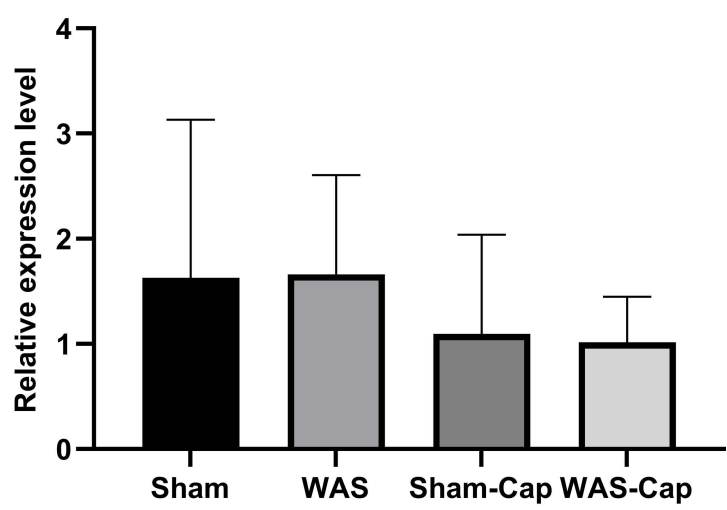

C
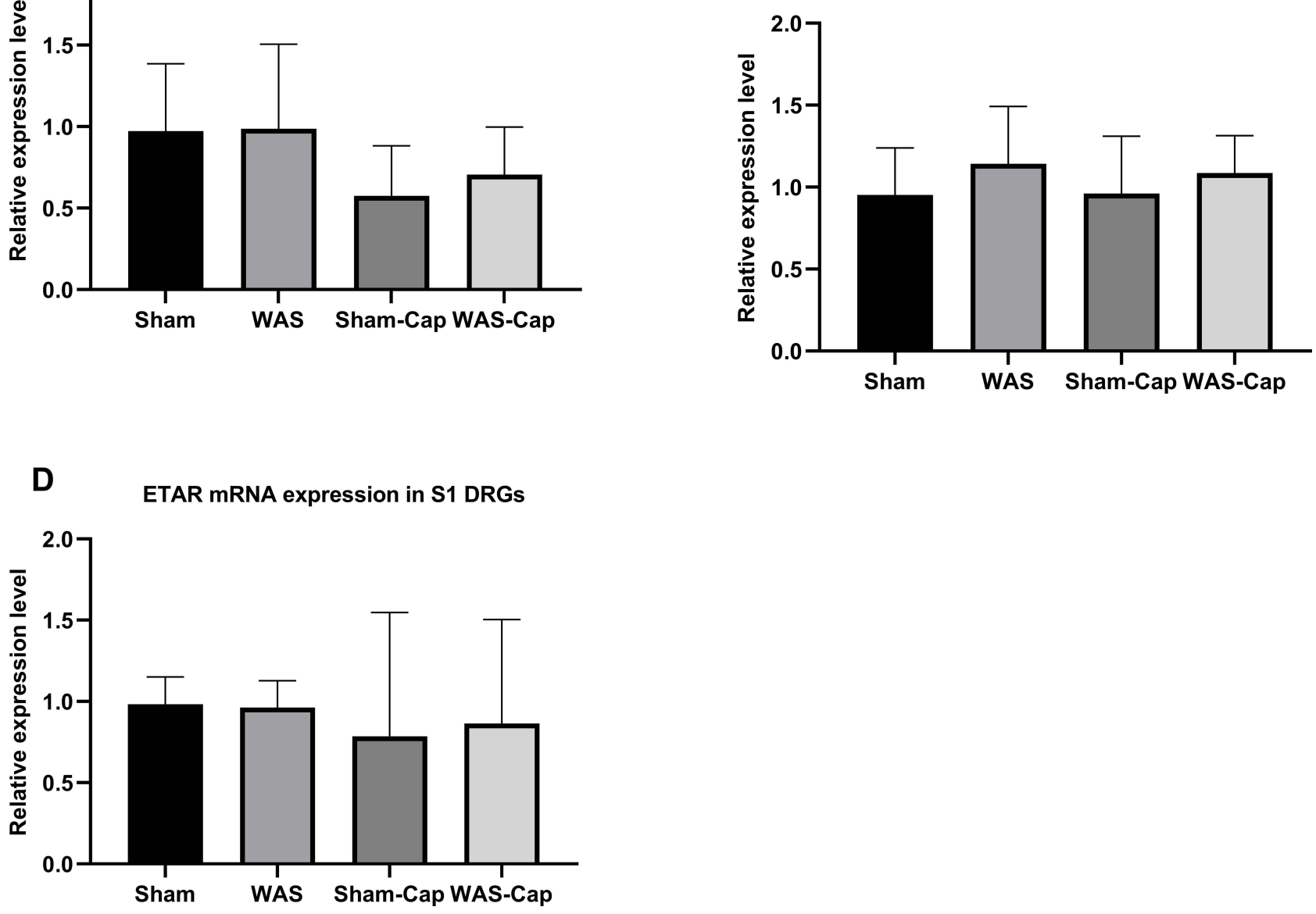

Figure 6 The expression of endothelin receptor type A (ETAR) in the bladder and L6-SI dorsal root ganglions (DRGs). (A) Relative expression of ETAR protein in bladder tissue. (B) Relative expression of ETAR mRNA in bladder tissue. (C) Relative expression of ETAR mRNA in L6 DRGs. (D) Relative expression of ETAR mRNA in SI DRGs. GAPDH, reduced glyceraldehyde-phosphate dehydrogenase.

these two groups. Thus, capsaicin administration diminished visceral hyperexcitability and attenuated many stress-related functional changes, revealing the role of C-fiber afferents in the development of WAS-induced bladder dysfunction. However, mechanisms underlying this C-fiber afferent hyperexcitability remain unclear. So far, several candidate receptors have been identified as contributing to stress-induced bladder afferent hyperexcitability, including protease activated receptor, ${ }^{39}$ transient receptor potential channels (TRPV1,TRPV4, ${ }^{43,45}$ TRPA1 ${ }^{39}$ ), $\alpha 1$-adrenoceptor ${ }^{46}$ and 

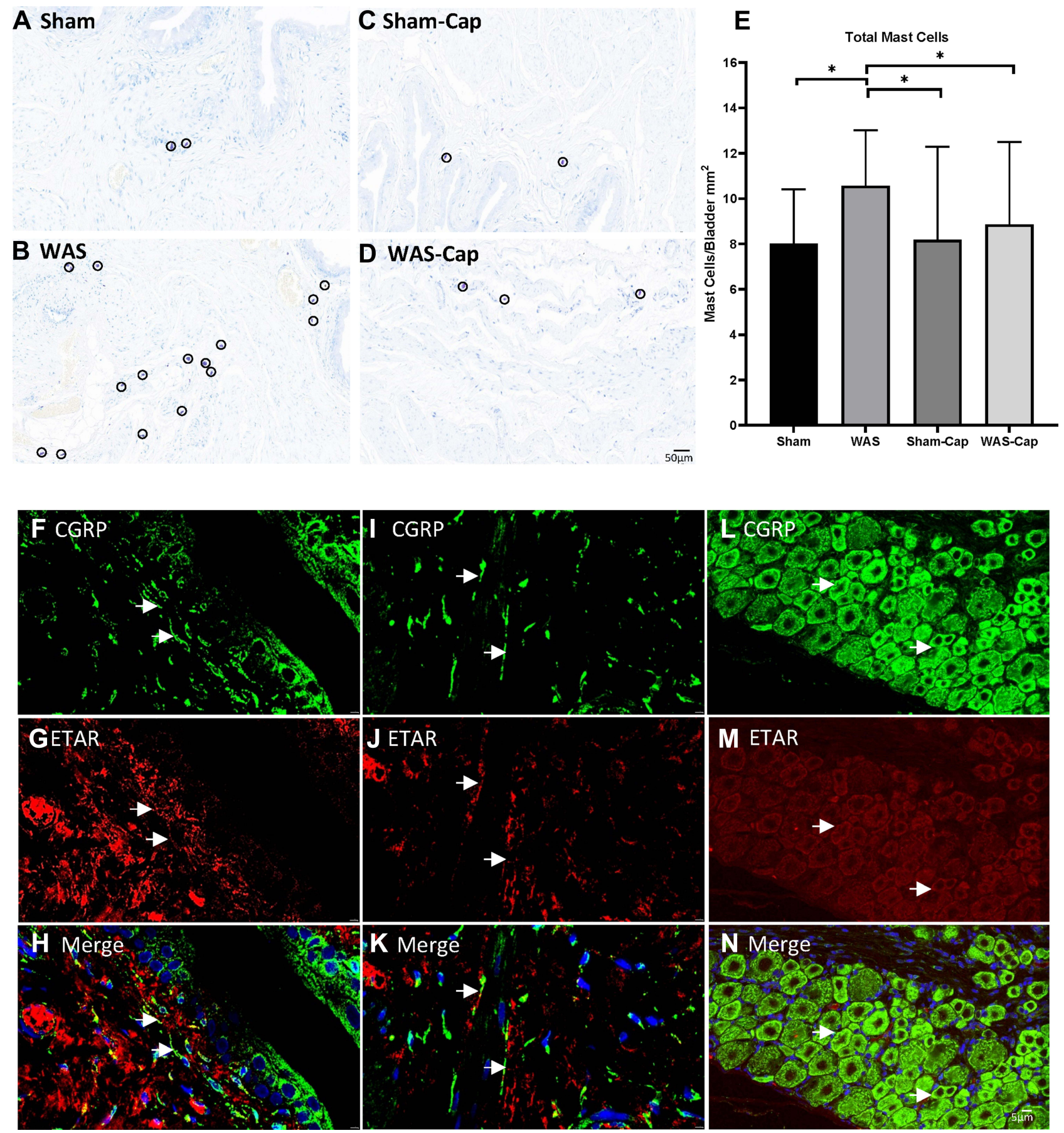

Figure 7 The toluidine blue staining and immunofluorescence staining of bladder and dorsal root ganglions (DRGs). (A-E) Representative toluidine blue staining of each group. Water avoidance appeared to show increased total mast cells, which could be attenuated by capsaicin pretreatment. (F-H) The immunoreactivity of endothelin receptor type A (ETAR, red) was positive and co-expressed with calcitonin gene-related peptides (CGRP, green) in the bladder mucosa of non-capsaicin-pretreated rats. (I-K) The immunoreactivity of ETAR was positive and co-expressed with CGRP) in the bladder muscle of non-capsaicin-pretreated rats. (L-N) The immunoreactivity of ETAR was positive and co-expressed with CGRP) in the L6 DRG of non-capsaicin-pretreated rats. Calibration bar was $20 \mu \mathrm{m}$ and $5 \mu \mathrm{m}$ in histological staining and immunostaining, respectively. *Represents $p<0.05$.

corticotropin releasing-factor receptor. ${ }^{47}$ Given the heterogeneity and intractability of IC/BPS, other mechanisms appear to be involved in stress-related bladder dysfunction and worthy of further exploration.

As mentioned above, ET-1 can bind to specific receptors to exert multiple effects on bladder functions such as inducing detrusor overactivity and modulating sensory function in the peripheral nervous system. ${ }^{25,27,48,49}$ This study 


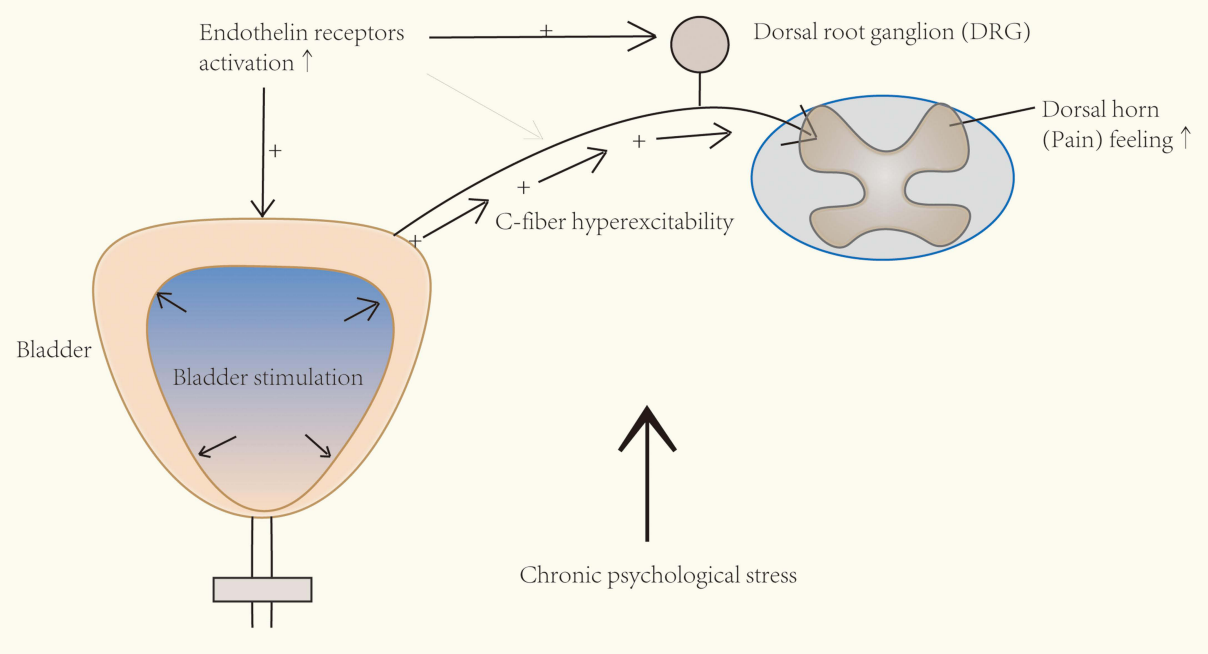

Figure 8 Schematic drawing of the involvement of endothelin pathway in chronic psychological stress-induced bladder hyperalgesia. After chronic stress exposure, the increased activation of endothelin receptors (possibly subtype A) may enhance the afferent input and in turn cause increased pain feeling.

also revealed the key role of endothelin pathway in the induction of bladder hypersensitization and hyperalgesia. In comparison with saline infusion, ET-1 infusion further significantly decreased the pressure to trigger PT and VMR, together with a more forceful and longer VMR. These results indicated that ET-1 administration could strengthen the effects of WAS on afferent sensory pathway, causing a further reduction in pain threshold but a further increased and prolonged pain input during bladder filling. In other words, binding of ET-1 to its receptors boosted the excitability of bladder afferents following WAS exposure, namely potentiation effect of endothelin pathway on WAS. This finding was in parallel with previous studies, which revealed that endothelin pathway could potentiate nociception induced by other algogens such as formalin, serotonin, and capsaicin. ${ }^{50}$

More importantly, we confirmed that endothelin pathway-potentiated effect on WAS exposure was resulted from the activation of endothelin receptors in capsaicin-sensitive bladder C-fiber afferents. In present study, ET-1 infusion induced nonsignificant difference in PT or VMR between WAS-Cap and Sham-Cap groups. These elucidated that capsaicin pretreatment attenuated the effects of ET-1 infusion by C-fiber desensitization, ignoring the existence of WAS exposure. Accordingly, ET-1 receptors may be implicated in the bladder sensory transduction mediated by C-fibers. ETAR, rather than ETBR, was apparently involved in this ET-1-mediated sensory transduction due to the following evidences. For anatomical locations, ETAR immunoreactivity in rats was presented in a subset of small C-fiber sensory DRG neurons and their axons, while ETBR immunoreactivity was detected in DRG satellite cells and Schwann cells. ${ }^{51,52}$ For expression level, ETAR displayed predominant density than ETBR in particular bladder tissues ${ }^{53}$ and ET-1 showed the greatest affinity to ETAR. ${ }^{54}$ For physiological functions, ETAR activation in the periphery mostly provoked pain or nociceptive sensitization, while the effects attributed to ETBR were usually anti-nociceptive. ${ }^{55}$ Therefore, we checked the expression of ETAR in both bladder and DRGs. Unfortunately, no significant difference in ETAR expression was found between all four groups, possibly related to the limited number of rats or the relatively short period of WAS exposure. So WAS exposure may sensitize ETAR to ET-1 and lead to the following bladder dysfunctions. Immunostaining results confirmed the coexistence of ETAR and CGRP in bladder and DRGs, suggestive of the location of ETAR in C-fiber afferents. Similar studies also documented ET-1 receptors mediating peripheral pain signaling via C-fiber afferents (in review). ${ }^{27}$ Moreover, ETAR could sensitize TRPV1 and TRPA1 signaling via C-fiber afferents, ${ }^{28}$ and both TRPV1 and TRPA1 may participate the development of IC/BPS. ${ }^{36,56,57}$

In addition, two interesting results were found during CMG and VMR analysis. One was the different profile features between first voiding circle and continuous ones. During saline infusion, a relatively lower PT and VMR threshold/ IVPmax ratio were presented in continuous voiding cycles other than the first one, suggesting the facilitation effect of 
continuous infusion on micturition reflex. The existed residual volume after prior voiding phase may contribute to this effect. The other one was the capsaicin-induced decrease in IVPmax during both saline and ET-1 infusion. This difference may be related to the reduction in afferent input and subsequent the lack of efferent inhibition, resulting in reflex detrusor contraction as a reaction on muscle stretch (due to filling) at low volume. It was, to a certain degree, similar to the bladder dysfunction in neurogenic bladder due to spinal cord injury ${ }^{58}$ or underactive bladder due to bilateral pelvic nerve injury. ${ }^{59}$

Several postulated mechanisms may underpin the activation of endothelin receptors in bladder C-fiber afferents after WAS exposure. Firstly, WAS-induced inflammatory alternations may activate endothelin receptors. Robust studies have demonstrated the linking between inflammation and C-fiber (see in review), ${ }^{22}$ as well as the interaction between inflammation and the ET-1/ETAR pathway. ${ }^{31,32,60}$ Our group previously showed profound inflammatory alterations in the bladders of WAS rats. ${ }^{7}$ Increased total mast cells here also partly confirmed inflammation in WAS rats, which was seemingly reversed by capsaicin pretreatment. Secondly, WAS exposure may directly result in peripheral endothelin system dysfunction via ETAR pathway. Convincing evidence suggested that brief episodes of mental stress caused a chronic endothelial dysfunction, which was prevented by selective ETAR antagonism. ${ }^{33}$ Early life stress also produced alterations in endothelin pathway. ${ }^{34}$ In our study, a 10-day WAS exposure could, at least in part, increase the expression of ETAR or sensitize endothelin receptor to its ligands. Thirdly, strain-specific difference might influence the activation of endothelin receptors after WAS exposure. As reported by Robbins et al, chronic stress significantly increased bladder nociceptive responses only in high-anxiety rats (WKY) rather than Sprague-Dawley rats. ${ }^{61}$ Moreover, WKY rats showed a greater magnitude of the bladder contraction response to ET-1 versus SpragueDawley rats. ${ }^{49}$ Further studies are needed to determine the definite pertinent factors to activate endothelin pathway after WAS.

However, certain limitations should be mentioned. Above all, CMG and VMR recording from urethane anesthetized rats may not always be extrapolated to conscious rats. Additional analysis under conscious condition seem to be necessary, such as metabolic cage study and conscious-filling cystometry. Besides, although ETAR was conceivably involved in WAS-induce bladder dysfunction due to aforementioned findings, no sufficient evidence could directly support it. Moreover, we did not perform quantitative comparison of bladder inflammation level of other inflammatory markers before and after capsaicin pretreatment. Our previous study noted no evidence for an increase in inflammatory markers in harvested WAS bladder tissues. ${ }^{7}$ Lastly, we should admit that individual differences likely existed in CMG and VMR recording, partly reducing the confidence power. However, the main goal of this study was to report our initial findings that endothelin pathway may be functionally involved in chronic stress-induced bladder dysfunction through C-fiber afferents. One strength of our study was the utilization of a novel IC/BPS animal model with a comprehensive in vivo functional analysis. We also investigated the CMG recording during the first voiding and continuous voiding, respectively. Despite the nature of instability, the first voiding could mimic a physiological micturition and serve as a supplement. Moreover, the alternations in DRGs were also enrolled for analysis. Sometimes harvesting DRGs and performing analysis such qRT-PCR remain a considerable challenge. Our findings added a piece of important information for a better understanding the interplay between stress and bladder dysfunction.

\section{Conclusion}

Our study firstly reported that the activation of ET-1 receptors, possibly ETAR subtype, could increase chronic psychological stress-induced bladder hypersensitization and hyperalgesia through capsaicin-sensitive C-fiber afferents. This work shed some lights on the mechanistic pathways linking chronic stress to IC/PBS. Targeting peripheral ET-1 receptors could be an effective treatment for IC/BPS. Further studies are needed to clarify the involved receptor type of ET-1, delineate the possible mechanisms and identify the potential therapeutic drugs.

\section{Data Sharing Statement}

All data generated in this study are included in this manuscript. 


\section{Acknowledgments}

This study was supported by the grants from National Natural Science Foundation of China (No. 81800669) and from Natural Science Foundation of Hunan Province (No. 2021JJ40829).

\section{Author Contributions}

CY Qin: Conceptualization, Methodology, Formal analysis, Writing-original draft; YH Wang: Methodology, Supervision; S Li, Methodology, Resources; YY Tang: Formal analysis; YL Gao, Methodology, Investigation, Resources, Supervision, Project administration, Writing- review and editing. All authors made substantial contributions to conception and design, acquisition of data, or analysis and interpretation of data; took part in drafting the article or revising it critically for important intellectual content; agreed to submit to the current journal; gave final approval of the version to be published; and agree to be accountable for all aspects of the work.

\section{Disclosure}

The authors report no conflicts of interest in this work.

\section{References}

1. Hanno P, Erickson D, Moldwin R, Faraday M. Diagnosis and treatment of interstitial cystitis/bladder pain syndrome: AUA guideline amendment. J Urol. 2015;193(5):1545-1553. doi:10.1016/j.juro.2015.01.086

2. Konkle KS, Berry SH, Elliott MN, et al. Comparison of an interstitial cystitis/bladder pain syndrome clinical cohort with symptomatic community women from the RAND interstitial cystitis epidemiology study. J Urol. 2012;187(2):508-512. doi:10.1016/j.juro.2011.10.040

3. Suskind AM, Berry SH, Ewing BA, Elliott MN, Suttorp MJ, Clemens JQ. The prevalence and overlap of interstitial cystitis/bladder pain syndrome and chronic prostatitis/chronic pelvic pain syndrome in men: results of the RAND interstitial cystitis epidemiology male study. $J$ Urol. 2013;189 (1):141-145. doi:10.1016/j.juro.2012.08.088

4. Rothrock NE, Lutgendorf SK, Kreder KJ, Ratliff T, Zimmerman B. Stress and symptoms in patients with interstitial cystitis: a life stress model. Urology. 2001;57(3):422-427. doi:10.1016/S0090-4295(00)00988-2

5. Coyne KS, Kaplan SA, Chapple CR, et al. Risk factors and comorbid conditions associated with lower urinary tract symptoms: epiLUTS. BJU Int. 2009;103(Suppl 3):24-32. doi:10.1111/j.1464-410X.2009.08438.x

6. Griffith JW, Stephens-Shields AJ, Hou X, et al. Pain and urinary symptoms should not be combined into a single score: psychometric findings from the MAPP research network. $J$ Urol. 2016;195(4 Pt 1):949-954. doi:10.1016/j.juro.2015.11.012

7. Smith AL, Leung J, Kun S, et al. The effects of acute and chronic psychological stress on bladder function in a rodent model. Urology. 2011;78 (4):967 e961-967. doi:10.1016/j.urology.2011.06.041

8. Lee UJ, Ackerman AL, Wu A, et al. Chronic psychological stress in high-anxiety rats induces sustained bladder hyperalgesia. Physiol Behav. 2015;139:541-548. doi:10.1016/j.physbeh.2014.11.045

9. Ackerman AL, Jellison FC, Lee UJ, Bradesi S, Rodriguez LV. The Glt1 glutamate receptor mediates the establishment and perpetuation of chronic visceral pain in an animal model of stress-induced bladder hyperalgesia. Am J Physiol Renal Physiol. 2016;310(7):F628-F636. doi:10.1152/ ajprenal.00297.2015

10. Gao Y, Zhang R, Chang HH, Rodriguez LV. The role of C-fibers in the development of chronic psychological stress induced enhanced bladder sensations and nociceptive responses: a multidisciplinary approach to the study of urologic chronic pelvic pain syndrome (MAPP) research network study. Neurourol Urodyn. 2017;37(2):673-680. doi:10.1002/nau.23374

11. Sanford MT, Yeh JC, Mao JJ, et al. Voluntary exercise improves voiding function and bladder hyperalgesia in an animal model of stress-induced visceral hypersensitivity: a multidisciplinary approach to the study of urologic chronic pelvic pain syndrome research network study. Neurourol Urodyn. 2020;39(2):603-612. doi:10.1002/nau.24270

12. Wang Z, Chang HH, Gao Y, et al. Effects of water avoidance stress on peripheral and Central responses during bladder filling in the rat: a multidisciplinary approach to the study of urologic chronic pelvic pain syndrome (MAPP) research network study. PLoS One. 2017;12(9): e0182976. doi:10.1371/journal.pone.0182976

13. Cetinel S, Ercan F, Cikler E, Contuk G, Sener G. Protective effect of melatonin on water avoidance stress induced degeneration of the bladder. J Urol. 2005;173(1):267-270. doi:10.1097/01.ju.0000145891.35810.56

14. Saglam B, Cikler E, Zeybek A, Cetinel S, Sener G, Ercan F. An aqueous garlic extract alleviates water avoidance stress-induced degeneration of the urinary bladder. BJU Int. 2006;98(6):1250-1254. doi:10.1111/j.1464-410X.2006.06511.x

15. Okasha EF, Bayomy NA. Protective role of quercetin (Phytochemical) against water avoidance stress induced interstitial cystitis of adult female albino rats light and electron microscopic study. Egypt J Histol. 2010;33:649-658.

16. Holschneider DP, Wang Z, Chang H, et al. Ceftriaxone inhibits stress-induced bladder hyperalgesia and alters cerebral micturition and nociceptive circuits in the rat: a multidisciplinary approach to the study of urologic chronic pelvic pain syndrome research network study. Neurourol Urodyn. 2020;39(6):1628-1643. doi:10.1002/nau.24424

17. Zeybek A, Saglam B, Cikler E, Cetinel S, Ercan F, Sener G. Taurine ameliorates stress-induced degeneration of the urinary bladder. Acta Histochem. 2007;109(3):208-214. doi:10.1016/j.acthis.2006.12.001

18. Bazi T, Hajj-Hussein IA, Awwad J, Shams A, Hijaz M, Jurjus A. A modulating effect of epigallocatechin gallate (EGCG), a tea catechin, on the bladder of rats exposed to water avoidance stress. Neurourol Urodyn. 2013;32(3):287-292. doi:10.1002/nau.22288 
19. Yamamoto K, Takao T, Nakayama J, et al. Water avoidance stress induces frequency through cyclooxygenase-2 expression: a bladder rat model. Int J Urol. 2012;19(2):155-162. doi:10.1111/j.1442-2042.2011.02905.x

20. Matos R, Serrao P, Rodriguez L, Birder LA, Cruz F, Charrua A. The water avoidance stress induces bladder pain due to a prolonged alpha1A adrenoceptor stimulation. Naunyn Schmiedebergs Arch Pharmacol. 2017;390(8):839-844. doi:10.1007/s00210-017-1384-1

21. Kullmann FA, McDonnell BM, Wolf-Johnston AS, et al. Stress-induced autonomic dysregulation of mitochondrial function in the rat urothelium. Neurourol Urodyn. 2019;38(2):572-581. doi:10.1002/nau.23876

22. Yoshimura N, Oguchi T, Yokoyama H, et al. Bladder afferent hyperexcitability in bladder pain syndrome/interstitial cystitis. Int J Urol. 2014;21 (Suppl 1):18-25. doi:10.1111/iju.12308

23. Arms L, Vizzard MA. Neuropeptides in lower urinary tract function. Handb Exp Pharmacol. 2011;2011(202):395-423.

24. Ukai M, Yuyama H, Noguchi Y, et al. Participation of endogenous endothelin and ETA receptor in premicturition contractions in rats with bladder outlet obstruction. Naunyn Schmiedebergs Arch Pharmacol. 2006;373(3):197-203. doi:10.1007/s00210-006-0064-3

25. Ogawa T, Kamo I, Pflug BR, et al. Differential roles of peripheral and spinal endothelin receptors in the micturition reflex in rats. $J$ Urol. 2004;172 (4 Pt 1):1533-1537. doi:10.1097/01.ju.0000139540.56916.0e

26. Cartwright R, Franklin L, Tikkinen KAO, et al. Genome-wide association study identifies two novel loci associated with female stress and urgency urinary incontinence. J Urol. 2021;206(3):679-687.

27. Smith TP, Haymond T, Smith SN, Sweitzer SM. Evidence for the endothelin system as an emerging therapeutic target for the treatment of chronic pain. J Pain Res. 2014;7:531-545. doi:10.2147/JPR.S65923

28. Wu L, Liu TT, Jin Y, Wei S, Qiu CY, Hu WP. Endothelin-1 enhances acid-sensing ion channel currents in rat primary sensory neurons. Acta Pharmacol Sin. 2020;41(8):1049-1057. doi:10.1038/s41401-019-0348-Z

29. Zhou Z, Davar G, Strichartz G. Endothelin-1 (ET-1) selectively enhances the activation gating of slowly inactivating tetrodotoxin-resistant sodium currents in rat sensory neurons: a mechanism for the pain-inducing actions of ET-1. J Neurosci. 2002;22(15):6325-6330. doi:10.1523/ JNEUROSCI.22-15-06325.2002

30. Feng B, Strichartz G. Endothelin-1 raises excitability and reduces potassium currents in sensory neurons. Brain Res Bull. 2009;79(6):345-350. doi:10.1016/j.brainresbull.2009.04.012

31. Xie H, Wang H, Wu Q, et al. Endothelin-1/Endothelin receptor type A-Angiopoietins/Tie-2 pathway in regulating the cross talk between glomerular endothelial cells and podocytes in trichloroethylene-induced renal immune injury. J Inflamm Res. 2021;14:761-776. doi:10.2147/JIR.S301104

32. Bajory Z, Hutter J, Krombach F, Messmer K. The role of endothelin-1 in ischemia-reperfusion induced acute inflammation of the bladder in rats. J Urol. 2002;168(3):1222-1225. doi:10.1016/S0022-5347(05)64629-0

33. Spieker LE, Hurlimann D, Ruschitzka F, et al. Mental stress induces prolonged endothelial dysfunction via endothelin-A receptors. Circulation. 2002;105(24):2817-2820. doi:10.1161/01.CIR.0000021598.15895.34

34. Loria AS, D'Angelo G, Pollock DM, Pollock JS. Early life stress downregulates endothelin receptor expression and enhances acute stress-mediated blood pressure responses in adult rats. Am J Physiol Regul Integr Comp Physiol. 2010;299(1):R185-191. doi:10.1152/ajpregu.00333.2009

35. Pare WP. Learning behavior, escape behavior, and depression in an ulcer susceptible rat strain. Integr Psychol Behav Sci. 1992;27(2):130-141. doi:10.1007/BF02698502

36. Du S, Araki I, Yoshiyama M, Nomura T, Takeda M. Transient receptor potential channel A1 involved in sensory transduction of rat urinary bladder through C-fiber pathway. Urology. 2007;70(4):826-831. doi:10.1016/j.urology.2007.06.1110

37. Park JS, Jin MH, Hong CH. Neurologic mechanisms underlying voiding dysfunction due to prostatitis in a rat model of nonbacterial prostatic inflammation. Int Neurourol J. 2018;22(2):90-98. doi:10.5213/inj.1836124.062

38. Ercan F, Cetinel S, Erin N, et al. Volume of nerve fibers in the stress-induced bladder of adult rats following capsaicin treatment. Urol Int. 2003;71 (4):393-398. doi:10.1159/000074093

39. Pierce AN, Di Silvestro ER, Eller OC, Wang R, Ryals JM, Christianson JA. Urinary bladder hypersensitivity and dysfunction in female mice following early life and adult stress. Brain Res. 2016;1639:58-73. doi:10.1016/j.brainres.2016.02.039

40. Million M, Wang L, Wang Y, et al. CRF2 receptor activation prevents colorectal distension induced visceral pain and spinal ERK1/2 phosphorylation in rats. Gut. 2006;55(2):172-181. doi:10.1136/gut.2004.051391

41. Chang HH, Havton LA. Modulation of the visceromotor reflex by a lumbosacral ventral root avulsion injury and repair in rats. Am J Physiol Renal Physiol. 2012;303(5):F641-647. doi:10.1152/ajprenal.00094.2012

42. Alrashdi SF, Deliyanti D, Wilkinson-Berka JL. Intravitreal administration of endothelin type A receptor or endothelin type B receptor antagonists attenuates hypertensive and diabetic retinopathy in rats. Exp Eye Res. 2018;176:1-9. doi:10.1016/j.exer.2018.06.025

43. Mingin GC, Heppner TJ, Tykocki NR, Erickson CS, Vizzard MA, Nelson MT. Social stress in mice induces urinary bladder overactivity and increases TRPV1 channel-dependent afferent nerve activity. Am J Physiol Regul Integr Comp Physiol. 2015;309(6):R629-638. doi:10.1152/ ajpregu.00013.2015

44. Chancellor MB, de Groat WC. Intravesical capsaicin and resiniferatoxin therapy: spicing up the ways to treat the overactive bladder. $J$ Urol. 1999;162(1):3-11. doi:10.1097/00005392-199907000-00002

45. Merrill L, Vizzard MA. Intravesical TRPV4 blockade reduces repeated variate stress-induced bladder dysfunction by increasing bladder capacity and decreasing voiding frequency in male rats. Am J Physiol Regul Integr Comp Physiol. 2014;307(4):R471-480. doi:10.1152/ ajpregu.00008.2014

46. Hattori T, Sugaya K, Nishijima S, Kadekawa K, Ueda T, Yamamoto H. Emotional stress facilitates micturition reflex: possible inhibition by an $\alpha 1$-adrenoceptor blocker in the conscious and anesthetized state. Int Neurourol J. 2019;23(2):100-108. doi:10.5213/inj.1836284.142

47. Fuentes IM, Pierce AN, Di Silvestro ER, Maloney MO, Christianson JA. Differential influence of early life and adult stress on urogenital sensitivity and function in male mice. Front Syst Neurosci. 2017;11:97. doi:10.3389/fnsys.2017.00097

48. Scheepe JR, van den Hoek J, Jünemann KP, Alken P. Endothelin-A-receptor antagonist LU 302146 inhibits electrostimulation-induced bladder contractions in vivo. Neurourol Urodyn. 2006;25(5):468-472. doi:10.1002/nau.20257

49. Westfall TD, McCafferty GP, Pullen M, et al. Effect of endothelin on bladder contraction: potential role in bladder hyperactivity. Pharmacology. 2003;69(1):7-11. doi:10.1159/000071243

50. Piovezan AP, D’Orléans-Juste P, Tonussi CR, Rae GA. Endothelins potentiate formalin-induced nociception and paw edema in mice. Can J Physiol Pharmacol. 1997;75(6):596-600. doi:10.1139/y97-057 
51. Pomonis JD, Rogers SD, Peters CM, Ghilardi JR, Mantyh PW. Expression and localization of endothelin receptors: implications for the involvement of peripheral glia in nociception. $J$ Neurosci. 2001;21(3):999-1006. doi:10.1523/JNEUROSCI.21-03-00999.2001

52. Tu NH, Katano T, Matsumura S, et al. $\mathrm{Na}(+) / \mathrm{K}(+)$-ATPase coupled to endothelin receptor type B stimulates peripheral nerve regeneration via lactate signalling. Eur J Neurosci. 2017;46(5):2096-2107. doi:10.1111/ejn.13647

53. Yokoyama Y, Osano A, Hayashi H, et al. Endothelin-1 receptors in rat tissues: characterization by bosentan, ambrisentan and CI-1020. Biol Pharm Bull. 2014;37(3):461-465. doi:10.1248/bpb.b13-00881

54. Arai H, Hori S, Aramori I, Ohkubo H, Nakanishi S. Cloning and expression of a cDNA encoding an endothelin receptor. Nature. 1990;348 (6303):730-732. doi:10.1038/348730a0

55. Barr TP, Kam S, Khodorova A, Montmayeur JP, Strichartz GR. New perspectives on the endothelin axis in pain. Pharmacol Res. 2011;63 (6):532-540. doi:10.1016/j.phrs.2011.02.002

56. DeBerry JJ, Schwartz ES, Davis BM. TRPA1 mediates bladder hyperalgesia in a mouse model of cystitis. Pain. 2014;155(7):1280-1287. doi:10.1016/j.pain.2014.03.023

57. Yang Y, Zhang H, Lu Q, et al. Suppression of adenosine A(2a) receptors alleviates bladder overactivity and hyperalgesia in cyclophosphamide-induced cystitis by inhibiting TRPV1. Biochem Pharmacol. 2021;183:114340. doi:10.1016/j.bcp.2020.114340

58. Rosier PF. Practice of urodynamics in patients with neurogenic lower urinary tract dysfunction. In: Neuro-Urology. Springer. 2018:163-179.

59. Deruyver Y, Weyne E, Dewulf K, et al. Intravesical activation of the cation channel TRPV4 improves bladder function in a rat model for detrusor underactivity. Eur Urol. 2018;74(3):336-345. doi:10.1016/j.eururo.2018.05.020

60. Fujii T, Yamasaki R, Kira JI. Novel neuropathic pain mechanisms associated with allergic inflammation. Front Neurol. 2019;10:1337. doi:10.3389/ fneur.2019.01337

61. Robbins M, DeBerry J, Ness T. Chronic psychological stress enhances nociceptive processing in the urinary bladder in high-anxiety rats. Physiol Behav. 2007;91(5):544-550. doi:10.1016/j.physbeh.2007.04.009

Journal of Inflammation Research

\section{Dovepress}

\section{Publish your work in this journal}

The Journal of Inflammation Research is an international, peer-reviewed open-access journal that welcomes laboratory and clinical findings on the molecular basis, cell biology and pharmacology of inflammation including original research, reviews, symposium reports, hypothesis formation and commentaries on: acute/chronic inflammation; mediators of inflammation; cellular processes; molecular mechanisms; pharmacology and novel anti-inflammatory drugs; clinical conditions involving inflammation. The manuscript management system is completely online and includes a very quick and fair peer-review system. Visit http://www.dovepress.com/testimonials.php to read real quotes from published authors.

Submit your manuscript here: https://www.dovepress.com/journal-of-inflammation-research-journal 